\title{
Stabilizing chaotic vortex trajectories: an example of high-dimensional control
}

\author{
Á. Péntek ${ }^{1}$, J. B. Kadtke ${ }^{1}$, and Z. Toroczkai ${ }^{2,3}$ \\ ${ }^{1}$ Institute for Pure and Applied Physical Sciences, University of California at San Diego, \\ 9500 Gilman Dr., La Jolla, CA 92093-0360 \\ ${ }^{2}$ Department of Physics, Virginia Polytechnic Institute and State University, \\ Blacksburg, VA 24061-0435 \\ ${ }^{3}$ Institute for Theoretical Physics, Eötvös University, \\ Puskin utca 5-7, H-1088 Budapest, Hungary
}

(October 7, 1996)

\begin{abstract}
A chaos control algorithm is developed to actively stabilize unstable periodic orbits of higher-dimensional systems. The method assumes knowledge of the model equations and a small number of experimentally accessible parameters. General conditions for controllability are discussed. The algorithm is applied to the Hamiltonian problem of point vortices inside a circular cylinder with applications to an experimental plasma system.
\end{abstract}




\section{INTRODUCTION}

Hamiltonian models of 3-D collinear vortex filaments or 2-D point vortices inside closed geometries have for many years attracted interest due to its central role in understanding the evolution of vorticity in real flows [1 6]. 2-D point vortex dynamics is also relevant in a series of real experimental applications such as vortices in superfluid helium [7], superconductors, and dynamics of non-neutral plasma filaments [8]. It has also been shown recently that point-vortex models can provide a good formulation for developing control algorithms in realistic flows [9], since they are able to capture in certain limits most of the qualitative features of the vorticity dynamics. These results indicate that control algorithms based on these low-dimensional point vortex models can be successfully implemented for certain geometries even in fully-viscid Navier-Stokes equations [10].

In this Letter we will likewise attempt to develop a low-dimensional model for a fluid control algorithm, aimed at modifying a realistic flow of vorticity inside a bounded fluid domain. The model will be based on Hamiltonian point vortex dynamics, and make use of unstable periodic trajectories in the natural flow dynamics to modify the flow via the Ott, Grebogi, Yorke (OGY) [11] scheme. The principal result here is that we develop a general control algorithm which can be utilized in higher-dimensional systems (phase space $D>3$ ), such as multi-vortex systems. We demonstrate the method's effectiveness using numerical examples that model an experimental system of confined non-neutral plasma.

To develop the control scheme, we first briefly review the formulation of point vortex dynamics. Let $z=x+i y$ denote the complex coordinate of a single vortex. The effect of the

boundary of the circular domain is simply accounted for by image charges at $R^{2} / z^{*}$. The Hamiltonian of a system of $N$ identical point vortices each of circulation $\Gamma$ inside a circle of radius $R$ is then given by [3]

$$
H\left(z_{1}, \ldots, z_{N}\right)=-\frac{\Gamma^{2}}{4 \pi}\left[\sum_{k \neq j}^{N} \ln \frac{\left|z_{k}-z_{j}\right|}{\left|R^{2}-z_{k} z_{j}^{*}\right|}-\sum_{k}^{N} \ln \left(R^{2}-\left|z_{k}\right|^{2}\right)\right],
$$

Each vortex moves by being passively advected in the velocity field created by the other 
vortices and the image vortices, with the dynamical equations being obtained from the canonical Hamilton relations:

$$
\Gamma \dot{z}_{k}^{*}=i \frac{\partial H}{\partial z_{k}}, \quad k=1, \ldots, N,
$$

that is

$$
\dot{z}_{k}^{*}=-\frac{i \Gamma}{4 \pi}\left[\sum_{j \neq k}^{N} \frac{1}{z_{k}-z_{j}}+\sum_{j}^{N} \frac{z_{j}^{*}}{R^{2}-z_{j}^{*} z_{k}}\right], \quad k=1, \ldots, N .
$$

Here, the first sum includes the velocity field created by the other vortices at the position of the $k$-th vortex (infinite self-interaction excluded) while the second sum gives the velocity field of the image vortices. The Hamilton relations (2) imply that each vortex contributes one degree-of-freedom and therefore two dimensions to the phase space. In addition, since the Hamiltonian does not contain time explicitly, the "energy" of the vortex system $E=$ $H\left(z_{1}, \ldots, z_{N}\right)$ is a constant of the motion. There exists another constant of motion for a circular domain resulting from the invariance of $H$ under rotation, i.e. the "angular momentum":

$$
L=\Gamma \sum_{k}^{N}\left|z_{k}\right|^{2} .
$$

Because of these two integrals of the motion, the dynamics of the $N$-vortex system is restricted to a $2 N-2$ dimensional manifold in the $2 N$ dimensional phase space.

The Hamilton relations for point vortices imply the unusual situation that the phase space is simply a re-scaled version of the configuration space. Hence, integrability conditions which normally rely on symmetries of the Hamiltonian via Noether's theorem, can partly be deduced by looking for symmetries in the physical domain [1] [3]. In this view, one has global non-integrability (chaos) if $N^{*} \geq 1$, where $N^{*}=$ (number of vortices) - (number of remaining integrals of motion) [四. For the circular domain discussed here, $N^{*} \geq 1$ for $N \geq 3$. In the following we will restrict our discussion to the three-vortex case, which is the smallest number of vortices inside a cylinder that leads to chaotic dynamics.

Our central aim here is to achieve control and avoid chaos by stabilizing simple unstable periodic orbits of the three-vortex system, thereby taking advantage of the natural dynamics 
of the flow. Although there are a large number of low-order periodic orbits of the generic vortex dynamics, we will restrict ourselves without loss of generality to those associated with symmetric configurations of vortices, with the center-of-vorticity in the origin. Examples of such configurations are shown in Fig. 1 for $N=3$. We can simplify the discussion by rendering the equations-of-motion dimensionless with the substitution:

$$
z \longrightarrow \frac{z}{R}, \quad \text { and } \quad t \longrightarrow t \frac{2 \pi}{\Gamma R^{2}}
$$

Performing a further transformation into a reference frame co-rotating with the vortex system with uniform angular velocity $\omega$, again without loss of generality, Eq. (3) will read as:

$$
\dot{z}_{k}^{*}=-\frac{i}{2}\left[\sum_{j \neq k}^{3} \frac{1}{z_{k}-z_{j}}+\sum_{j}^{3} \frac{z_{j}^{*}}{1-z_{j}^{*} z_{k}}-2 \omega z_{k}^{*}\right], \quad k=1,2,3 .
$$

The main advantage of this co-rotating frame is that some of the periodic orbits in the 6-D phase space reduce to a single fixed point (with proper choice of $\omega$ ).

The stability of such symmetric configurations has been first analyzed by Havelock [12]. In spite of the global non-integrability of the system, the symmetric orbits are stable for some parameter regimes, i.e. they are elliptic quasiperiodic orbits embedded in the fourdimensional manifold determined by the constants of motion (1) and (4). These orbits correspond to states where the vortices are away from the boundary of the cylinder, hence the effect of the "image" vortices is a weak perturbation, of the integrable three-vortex dynamics in free space. These regions are separated from the chaotic areas by KAM tori containing orbits of marginal stability. Many of these stable configurations have been found experimentally in the dynamics of magnetically confined non-neutral plasma columns [8], where the dynamics is very close to those of point vortices [13.

\section{FORMULATION OF A HIGH-DIMENSIONAL CONTROL ALGORITHM}

Although many examples of chaos control algorithms have been developed in recent years, these have been restricted to a relatively low-dimensional phase space. Here, we develop a 
control algorithm for higher-dimensional systems which assumes explicit knowledge of the dynamical equations and therefore the location, eigenvalues and eigenvectors of the fixed point or periodic orbit to be stabilized. This method can also certainly be reformulated in the language of experimentally measured time series (as in previous works [14] 16] ), however this is not our immediate aim here.

For the particular application we discuss in this Letter, we aim to modify the dynamics of the fully-viscid continuous fluid using a control model based on the point vortex dynamics. Hence, the model governing equations are known, and the high-dimensional control is straightforward to calculate. In the case of an experimental system, the method can be implemented in an identical fashion, using the Jacobian and the perturbation matrix obtained from experimental time-series [17].

To begin, let $\dot{\mathbf{r}}=\mathbf{A}(\mathbf{r}, \boldsymbol{\Phi})$ represent the $n=2 N$-dimensional unperturbed dynamical system of Eq. (6). Here, in addition to Eq. (3) we have introduced a set of $m$ experimentally accessible system parameters, represented by the $m$-dimensional vector $\boldsymbol{\Phi}$. We assume the number of such parameters $m$ is usually much smaller then the dimensionality of the phase space $n$, typically one.

Suppose that $\mathbf{r}_{0}$ is an unstable fixed point in the $n$-dimensional phase space, i.e., $\dot{\mathbf{r}}_{0}=\mathbf{A}\left(\mathbf{r}_{\mathbf{0}}, \Phi_{\mathbf{0}}\right)=0$, where $\boldsymbol{\Phi}_{\mathbf{0}}$ is a fixed set of parameters of interest. Allowing small perturbations $\delta \boldsymbol{\Phi}(t)$ in the parameters, then in a neighborhood of this fixed point $\mathbf{r}=\mathbf{r}_{0}+\delta \mathbf{r}$ the dynamics is described by the linearized equations

$$
\delta \dot{\mathbf{r}}(t)=\mathbf{J}\left(\mathbf{r}_{0}, \boldsymbol{\Phi}_{0}\right) \delta \mathbf{r}(t)+\mathbf{G}\left(\mathbf{r}_{0}, \Phi_{0}\right) \delta \boldsymbol{\Phi}(t)
$$

where $\mathbf{J}=\partial \mathbf{A} / \partial \mathbf{r}$ is the standard Jacobian and the $n \times m$ matrix $\mathbf{G}=\partial \mathbf{A} / \partial \boldsymbol{\Phi}$ describes the effect of small parameter perturbations on the system (i.e. the perturbation matrix).

A stability analysis can be performed around the fixed point $\mathbf{r}_{0}$, by studying the properties of the Jacobian, and typically can reveal a multitude of topologies. These topologies are defined by the set of eigenvalues of $\mathbf{J}$, which can be real, complex or zero. The instability of the fixed point implies that there is at least one eigenvalue $\lambda$ with $\operatorname{Re}(\lambda)>0$. Since 
the system is Hamiltonian ( $\operatorname{Tr} \mathbf{J}=0)$ there is also at least one eigenvalue with negative real part. Although there is no guarantee of purely real eigenvalues and eigenvectors, with a proper transformation one can eliminate the imaginary part for at least one of the stable eigenvalues (and the corresponding eigenvector) without changing the stability. In the following, we will suppose without loss of generality that there is at least one purely real negative eigenvalue $\lambda_{s}<0$ with a corresponding normalized, stable real eigenvector $\mathbf{e}_{s}$.

To achieve control of the vortex trajectories using small perturbations $\delta \boldsymbol{\Phi}$, we impose the condition that after a short time $\Delta t$ the trajectory has approached the fixed point, i.e. $|\delta \mathbf{r}(t+\Delta t)|<|\delta \mathbf{r}(t)|$. This can generally be accomplished in many different ways. One possible criteria is suggested by the low-dimensional chaos control of OGY [11], where the new point $\delta \mathbf{r}(t+\Delta t)$ is driven onto the stable manifold of the fixed point. Other possible choices are the self-locating (geometric control) method originally developed for low-dimensional chaos [18,19] or a method using a Newton algorithm developed for higherdimensional systems [20]. Here, we adopt the approach of OGY, i.e. we require that the trajectory lies on the stable manifold of the fixed point after a time $\Delta t$ [9]:

$$
\delta \mathbf{r}(t+\Delta t)=\alpha|\delta \mathbf{r}(t)| \mathbf{e}_{s}
$$

where alpha is a small real number $(0<\alpha<1)$. Intuitively, this implies that after time $\Delta t$ the trajectory lies on the stable manifold and simultaneously the distance from the fixed point has been decreased by $\alpha$. Using a discretization of (可), this equation can be written explicitly as

$$
\alpha|\delta \mathbf{r}(t)| \mathbf{e}_{s} \simeq[\mathbf{1}+\mathbf{J} \Delta t] \delta \mathbf{r}(t)+\mathbf{G} \delta \mathbf{\Phi}(t) \Delta t
$$

where for simplicity the notations $\mathbf{J} \equiv \mathbf{J}\left(\mathbf{r}_{0}, \boldsymbol{\Phi}_{0}\right)$ and $\mathbf{G} \equiv \mathbf{G}\left(\mathbf{r}_{0}, \boldsymbol{\Phi}_{0}\right)$ have been introduced. This represents a system of $n$ linear equations with $m$ unknown perturbation parameters $\delta \Phi$ that typically has no solution when $m<n$. This means that it is not possible to control such a system with one or a few control parameters in a one-step process, as described by Eq. (9). 
Here, however we develop an alternative way to overcome this difficulty. Let us introduce the following notation for the RHS of Eq. (9):

$$
\mathbf{F}(\mathbf{r}, \boldsymbol{\Phi}) \equiv[\mathbf{1}+\mathbf{J} \Delta t] \delta \mathbf{r}(t)+\mathbf{G} \delta \boldsymbol{\Phi}(t) \Delta t
$$

Then, instead of Eq. (B) one can impose a weaker condition: namely, that after $p$ steps the trajectory lies on the stable manifold, i.e.

$$
\delta \mathbf{r}(t+p \Delta t)=\alpha|\delta \mathbf{r}(t)| \mathbf{e}_{s}
$$

or

$$
\alpha|\delta \mathbf{r}(t)| \mathbf{e}_{s}=\mathbf{F}\left(\mathbf{F}\left(\ldots\left(\mathbf{F}\left(\mathbf{r}, \delta \Phi^{(1)}\right), \ldots\right), \delta \boldsymbol{\Phi}^{(p-1)}\right), \delta \boldsymbol{\Phi}^{(p)}\right)
$$

where the shorthand notation $\delta \boldsymbol{\Phi}^{(\mathbf{k})}$ has been introduced for the perturbation at time $t+k \Delta t$, i.e. $\mathbf{\Phi}(t+k \Delta t)$. Eq (12) then can be written explicitly as

$$
\mathbf{M}^{p-1} \mathbf{G} \delta \boldsymbol{\Phi}^{(1)}+\mathbf{M}^{p-2} \mathbf{G} \delta \boldsymbol{\Phi}^{(2)}+\ldots+\mathbf{G} \delta \boldsymbol{\Phi}^{(p)}=\frac{1}{\Delta t}\left(\alpha|\delta \mathbf{r}(t)| \mathbf{e}_{s}-\mathbf{M}^{p} \delta \mathbf{r}(t)\right)
$$

where $\mathbf{M} \equiv \mathbf{1}+\mathbf{J} \Delta t$. This provides us with $n$ linear equations and $m p$ unknown perturbations. The number $p$ is choosen as the smallest integer satisfying the condition $m p \geq n$. In the following, we will assume that there is a $p$ such as $m p=n$. If $n$ is not divisible by $m$, there are $m p-n$ perturbation parameters that can be freely set to zero.

We note that the size $\delta r^{*}$ of the linear neighborhood of the fixed point gives a natural limit for the allowed size of the perturbations, and we assume that none of the perturbation parameters $\delta \Phi_{k}$ can be larger than a preselected value $\delta \Phi^{*}$. However, since Eq. (10) contains the yet unspecified control time parameter $\Delta t$, the perturbations scale with this parameter as $1 / \Delta t$. This means that we should impose a limit on the product $\delta \Phi_{k} \Delta t$ rather than on $\delta \Phi_{k}$ alone. The upper bound of this product should naturally be set by the system's physical limitations. For example, in the 3 -vortex problem $\Phi_{k} \Delta t$ is a length that is naturally limited by the typical size of the linearized region $\delta r^{*}$, i.e. $\delta \Phi^{*} \Delta t=\delta r^{*}$. 
It is possible to derive general conditions for the controllability of the system. To have a solution for the $p$-step control process (13), the square matrix $\mathbf{C}$ formed from the $p$ matrices of size $n \times m$, i.e.

$$
\mathbf{C} \equiv\left\{\mathbf{M}^{p-1} \mathbf{G} ; \mathbf{M}^{p-2} \mathbf{G} ; \ldots ; \mathbf{G}\right\}
$$

has to have nonvanishing determinant. In two-dimensions, the system is always controllable provided the vector $\mathbf{G}$ is not collinear with $\mathbf{e}_{s}$. Intuitively, this means that small perturbations of the system do not move the fixed point exclusively along the stable direction. In higher-dimensions, such a condition is not strong enough; the perturbation may access only a very limited subspace, or may have a certain symmetry that leads to un-controllability, even if the two-dimensional analog condition is satisfied. Instead

$$
\operatorname{det} \mathbf{C} \neq 0
$$

incorporates all these additional conditions, and can be viewed as the basic criteria of controllability. This relation is also a useful tool to select appropriate perturbations for each particular control problem, and to find the minimum number of perturbations that can successfully control the system. The control parameter selection is probably the most important step in any such problem, especially when the Jacobian is highly-symmetric as in the case of symmetric periodic orbits of identical vortices. For such orbits it is straightforward to show that most of the symmetric perturbations lead to un-controllability. An example of this is the simple perturbation of "squeezing" the boundary, i.e. making it slightly elliptic with a small eccentricity. Here, the equations of motion can be simply deduced by using a simple conformal mapping $f(z)=z+\epsilon z^{3}$ that maps the unit circle into an ellipse, with semi-axis $1+\epsilon$ and $1-\epsilon$, respectively (for $\epsilon \ll 1$ ). A careful analysis of the collinear configurations of Fig. 1(a) shows that the determinant of the corresponding controllability matrix $\mathbf{C}$ vanishes, due to the symmetry of the Jacobian $\mathbf{J}$ and the perturbation matrix $\mathbf{G}$ with respect to the non-central vortices. This means that it is not possible to control this particular configuration by using cylinder squeezing. In fact such behaviour is expected to be quite general in 
systems of interacting identical subsytems that respond in the very same way to external perturbations.

\section{NUMERICAL EXPERIMENTS}

To illustrate the above control algorithm, we actively stabilized the two simplest unstable periodic orbits of the three vortex system inside a cylinder, i.e. the symmetric states shown in Fig. 1(a,b). For the collinear state of Fig. 1(a), the angular velocity of the configuration is $\omega=\left(3+a^{4}\right) /\left(1-a^{4}\right) /\left(4 a^{2}\right)$, while for the triangular state of Fig. $1(\mathrm{~b})$, it is $\omega=\left(1+2 a^{6}\right) /(1-$ $\left.a^{6}\right) /\left(2 a^{2}\right)$. Here $a$ is the radial distance of the non-central vortices from the center of the cylinder. To simplify the equations of motion, the dynamics is viewed in a reference frame corotating with the vortices with this constant angular velocity $\omega$. In this frame, the periodic orbits become single fixed points. Analytic calculation of the Jacobian for the collinear state at $a=0.5$ reveals the existence of four real eigenvalues, $\lambda_{1,2}= \pm 4.7565$ and $\lambda_{3,4}=0$, and two purely complex ones, $\lambda_{5,6}=1.6027 i$. The triangular state at $a=0.7$ leads to two purely real eigenvalues $\lambda_{1,2}= \pm 0.0001165$ and four complex ones, $\lambda_{3,4,5,6}= \pm 0.6168 \pm 0.1670 i$. The stable eigendirection $\mathbf{e}_{s}$ is then obtained in each case as the eigenvector associated with the single negative real eigenvalue. One can observe that although the two topologies are completely different, the controller requires knowledge of only one of the possibly many stable directions.

To control the vortex dynamics we require non-symmetric and non-homogeneous perturbatios. We have introduced simple perturbations applied only at the cylinder surface: namely, a uniform distribution of sources and sinks with variable strength $s=s_{0} \cos (k \theta)$. Although such a perturbation may be difficult to implement in a real fluid experiment, it is a real possibility in the plasma analog of the vortex dynamics [8], where similar perturbations can be generated by external electric fields. For $k=1,2$, and 3 , this leads to a uniform, a quadrupole, and a sextapole field, respectively, as shown in Fig. 2(a-c). Since all these fields

are highly symmetric, none of them alone can effectively control the vortex dynamics. How- 
ever, their linear combinations, resulting in strongly asymmetric and inhomogenous fields, proved to be a successful choice that satisfies the controllability conditions (15). Here, we have introduceed three linearly independent external fields as shown in Fig. 2(d-f). Using these, the equations of motion can be written:

$$
\begin{aligned}
\dot{z}_{k}^{*}= & -\frac{i}{2}\left[\sum_{j \neq k}^{3} \frac{1}{z_{k}-z_{j}}+\sum_{j}^{3} \frac{z_{j}^{*}}{1-z_{j}^{*} z_{k}}-2 \omega z_{k}^{*}\right] \\
& +\Phi_{1}\left(1+z_{k}+z_{k}^{2}\right)+\Phi_{2}\left(-1+z_{k}-z_{k}^{2}\right)+\Phi_{3}\left(1+z_{k}-z_{k}^{2}\right) \quad k=1,2,3 .
\end{aligned}
$$

We previously showed that there are two constants of motion in the unperturbed system, the energy and the angular momentum. Due to the presence of the time-dependent perturbations that do not preserve these first integrals, however, the effective dynamics is no longer restricted to a 4-D manifold, but rather can occupy in the full 6-D phase space. Note that if the dynamics initially lies in the same energy and angular momentum manifold that contains our fixed point, ergodicity ensures that the trajectory sooner or later reaches a close neighborhood of the desired fixed point, and then the control algorithm is switched on.

If this is not the case, then in general this procedure can be supplemented with a targeting algorithm that first drives the system close to the proper energy and angular momentum values, then takes advantage of the infinite number of unstable periodic orbits in that manifold to reach the neighborhood of the desired fixed point in a short time [21]. The development of such a targeting algorithm in higher dimensions is clearly an important step that deserves further study.

Since this is not the primary emphasis of this Letter, in our numerical simulations we have started the system close to the unstable fixed point. To demonstrate this Fig. 3(a-b) shows the time evolution of a single coordinate pair $\left(x_{1}, y_{1}\right)$ of the vortex system in the collinear case, at first without control (up to mark A). Then, after the dynamics reaches a small neighborhood of the desired fixed point (mark B), the controller is switched on, and the trajectory is stabilized. Fig. 3(c-e) also shows the time evolution of the required perturbations. Clearly this demonstrates that the algorithm can effectively control the 
dynamics with only tiny perturbations applied on the boundary. For better visualization, Fig. 4 shows a three-dimensional projection of the phase space trajectory with and without control.

As previously mentioned, other control criteria can be used instead of the OGY method. For example, one can consider the high-dimensional control of $\mathrm{Xu}$ and Bishop, based on the Newton root finding algorithm [20]. This method does not assume knowledge of the stable direction, and it can be useful in cases where all eigenvalues are complex (with at least two having non-vanishing real part). Then, instead of condition (11) one can use the usual Newton root finding algorithm

$$
\mathbf{r}(t+p \Delta t)=\mathbf{r}(t)-\mathbf{J}^{-1}\left(\mathbf{r}_{0}\right) \mathbf{A}\left(\mathbf{r}_{\mathbf{0}}+\delta \mathbf{r}\right)
$$

However, this method fails when the Jacobian is not invertible. (We note here that the transition from Eq. (5) to Eq. (6) in Ref. [20] is mathematically incorrect, i.e. the solution (6) does not fulfill Eq. (5). Therefore the weaker condition (17) shown above should be used.)

In a realistic Navier-Stokes simulation our algorithm is effective on time scales significantly shorter than the viscous one $\left(R e^{1 / 2}\right)$. This is attractive since this time scale can be rather large in some plasma systems, where the viscosity is small and there are no boundarylayer effects due to the free-slip conditions on the boundary. Even in these circumstances, it should be noted that there is an additional non-viscous effect that is not present in the Hamiltonian model, the vortex merger due to the finite size of the vortices. Therefore effective control can be reached only with concentrated vortices that are far from each other, typically with $2 \rho / d \gtrsim 1.8$, where $d$ is the distance between vortices and $\rho$ the vortex radius computed according to Ref. [22]. Although this condition can be easily fulfilled while the controller is working, during the targeting algorithm or full chaotic dynamics the vortices may easily come close to each other. In this context, the principal usefulness of such a control scheme will be to prevent the vortex merger on short time scales from symmetric unstable initial conditions. 
In conclusion, we have demonstrated that the control algorithm derived in this Letter can effectively control higher-dimensional systems. We have also derived a controllability condition that does not depend on the particular control method used, since it contains only the Jacobian and the perturbation matrix as input parameters. In this sense, the result is quite general, and can be used to decide the type and number of perturbations needed to control a higher-dimensional system.

For the particular numerical example presented here, i.e. three-vortex dynamics inside a cylinder, the controller was designed in such a way that it could be implemented in a magnetically confined plasma experiment. At present, we also plan to implement the control algorithm in a realistic viscous fluid framework, and hence an effective targeting algorithm

has to be developed. In addition, in the present paper the controller was formulated using exactly known vortex coordinates. However, the control scheme itself can be entierly reformulated in a phase space reconstructed purely from wall signal (e.g. boundary pressure or voltage) measurements, thereby providing an algorithm which can be directly implemented in an experimental framework.

After the acceptance of this paper for publication it was brought to our attention a different formulation of the control algorithm that yields to a similar controlability matrix 23.

\section{ACKNOWLEDGEMENTS}

The authors wish to thank F. Driscoll, G. Pedrizzetti and T. Tél for very useful discussions. AP and JK wish to acknowledge partial support for this project by a grant from the Office of Naval Research, No. N00014-96-1-0056, U.S.-Hungarian Science and Technology Joint Fund under Project JFNo. 286 and 501, and by the Hungarian Science Foundation under Grant Nos. OTKA T17493, F17166. ZT wishes to acknowledge R.K.P. Zia and B. Schmittmann for their support and permanent encouragement. ZT has also been sponsored by the National Science Foundation through the Division of Material Research. 


\section{REFERENCES}

[1] E. A. Novikov, and Yu. B. Sedov, Sov. Phys. - JETP 48, 440 (1978).

[2] H. Aref, Phys. Fluids 22, 393 (1979).

[3] H. Aref, Ann. Rev. Fluid Mech. 15, 345 (1983).

[4] J. B. Kadtke, PhD Thesis, Brown University, (1987).

[5] L. Zannetti, and P. Franese, Euro. J. Mech. B/Fluids 12, 43 (1993).

[6] A. Pentek, T. Tel, and Z. Toroczkai, J. Phys. A 28, 2191 (1995); Fractals 3, 33 (1995).

[7] P. H. Roberts, and R. J. Donnelly, Ann. Rev. Fluid Mech. 6, 179 (1974).

[8] K. S. Fine, A. C. Cass, W. G. Flynn, and F. Driscoll, Phys. Rev. Lett. 75, 3277 (1995).

[9] J. B. Kadtke, A. Pentek, and G. Pedrizetti, Phys. Lett. A 204, 108 (1995).

[10] A. Pentek, J. B. Kadtke, and G. Pedrizzetti, Controlled capture of vortices in open viscous flows, preprint (1996).

[11] E. Ott, C. Grebogi, and J. A. Yorke, Phys. Rev. Lett. 64, 1196 (1990).

[12] T. H. Havelock, Phyl. Mag. S. 7 11, 617 (1931).

[13] R. H. Levy, Phys. Fluids 8, 1288 (1965).

[14] P. So, and E. Ott, Phys. Rev. E 51, 2955 (1995).

[15] M. Ding, et al., Phys. Rev. E 53, 4334 (1996).

[16] V. Petrov, E. Mihaliuk, S. K. Scott, and K. Showalter, Phys Rev. E 51, 3988 (1995).

[17] T. Shinbrot, C. Grebogi, E. Ott, and J. A. Yorke, Nature 363, 411 (1993).

[18] Z. Toroczkai, Phys. Lett. A 19071 (1994).

[19] B. Sass, and Z. Toroczkai, J. Phys. A 29, 3545 (1996). 
[20] D. Xu, and S. R. Bishop, Phys. Lett. A 210, 273 (1996).

[21] T. Shinbrot, et al., Phys. Rev. Lett. 68, 2863 (1992).

[22] K. S. Fine, C. F. Driscoll, J. H. Malmberg, and T. B. Mitchell, Phys. Rev. Lett. 67, 588 (1991).

[23] F. J. Romeiras, C. Grebogi, E. Ott, and W. P. Dayawansa, Physica D 58, 165 (1992). 


\section{FIGURES}

FIG. 1. Symmetric vortex configurations leading to periodic orbits. Three-vortex configurations: (a) collinear with one vortex in the center; (b) equilateral triangle. This configuration leads to stable dynamics for $a<0.567$, and unstable one for $a>0.567$. Each configuration is rotating with an angular velocity $\omega$ that depends on the particular configuration.

FIG. 2. Perturbation fields used to control the vortex dynamics. Figs. (a-c) show the velocity fields generated by a distribution of sources and sinks on the boundary of strength $\cos (k \theta)$ for $k=1,2,3$, respectively. Figs. (d-f) displays the velocity fields resulting from different linear combinations of the fields (a-c): (d) $\dot{z}^{*}=\Phi_{1}\left(1+z+z^{2}\right),(\mathrm{e}) \dot{z}^{*}=\Phi_{2}\left(-1+z-z^{2}\right)$, and (c) $\dot{z}^{*}=\Phi_{3}\left(1+z-z^{2}\right)$. The strength of these fields $\Phi_{k}, k=1,2,3$ are computed by the control algorithm.

FIG. 3. (a) Vortex trajectories in the lab frame and the applied perturbations. Figs. (a-b) show the coordinates of one of the vortices, with the collinear configuration started close to the fixed point. Control is off until time $t=20$ (mark A). At this point the controller is activated and control begins at mark B. Without a targeting algorithm, the time between $\mathrm{A}$ and $\mathrm{B}$ can be very long. From instant B, the controller applies the necessary perturbations at intermittent times $\Delta t=0.1$ to keep the trajectory on the unstable periodic orbit. The parameter $\alpha$ in Eq. (13) was 0.1 . Figs. (c-e) show the applied perturbations. After a short transient time the perturbations are quite small, invisible on this scale. Fig. (e), inset, also displays the perturbation $\Phi_{3}$ magnified 1000 times between $30<t<40$, for visualization.

FIG. 4. Three-dimensional projection $\left(x_{1}, y_{1}, r_{12}=\left|z_{2}-z_{1}\right|\right)$ of the phase space trajectory in the lab frame. (a) Uncontrolled trajectory of the collinear configuration. (b) Trajectory after controller is switched on. 

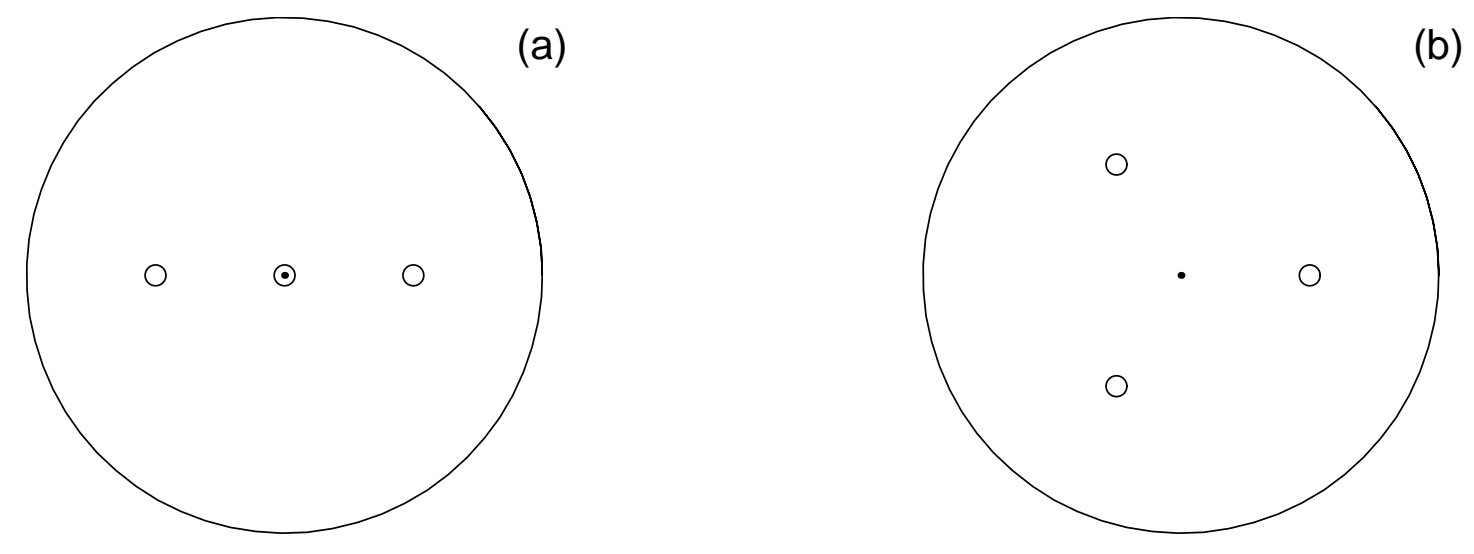

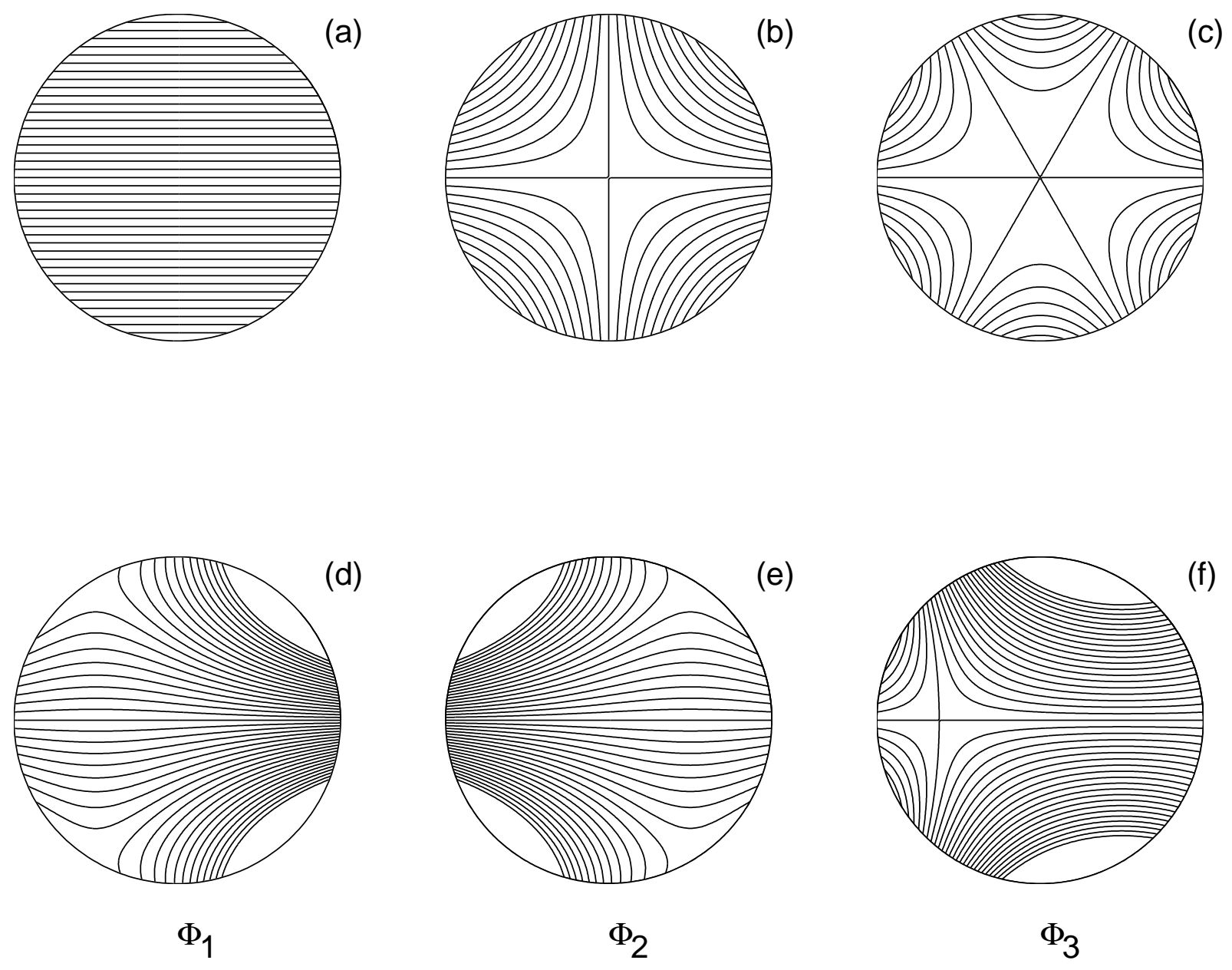

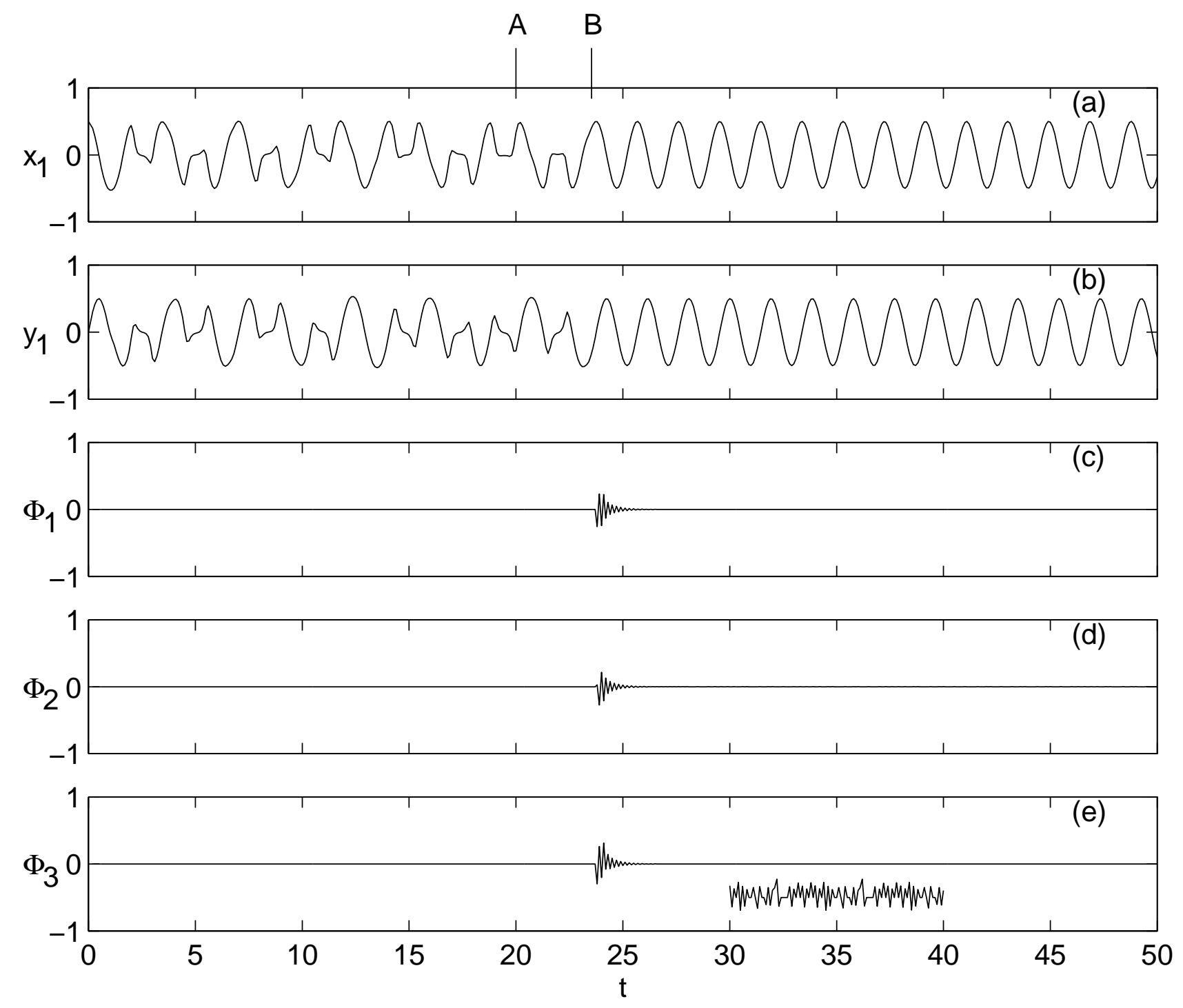

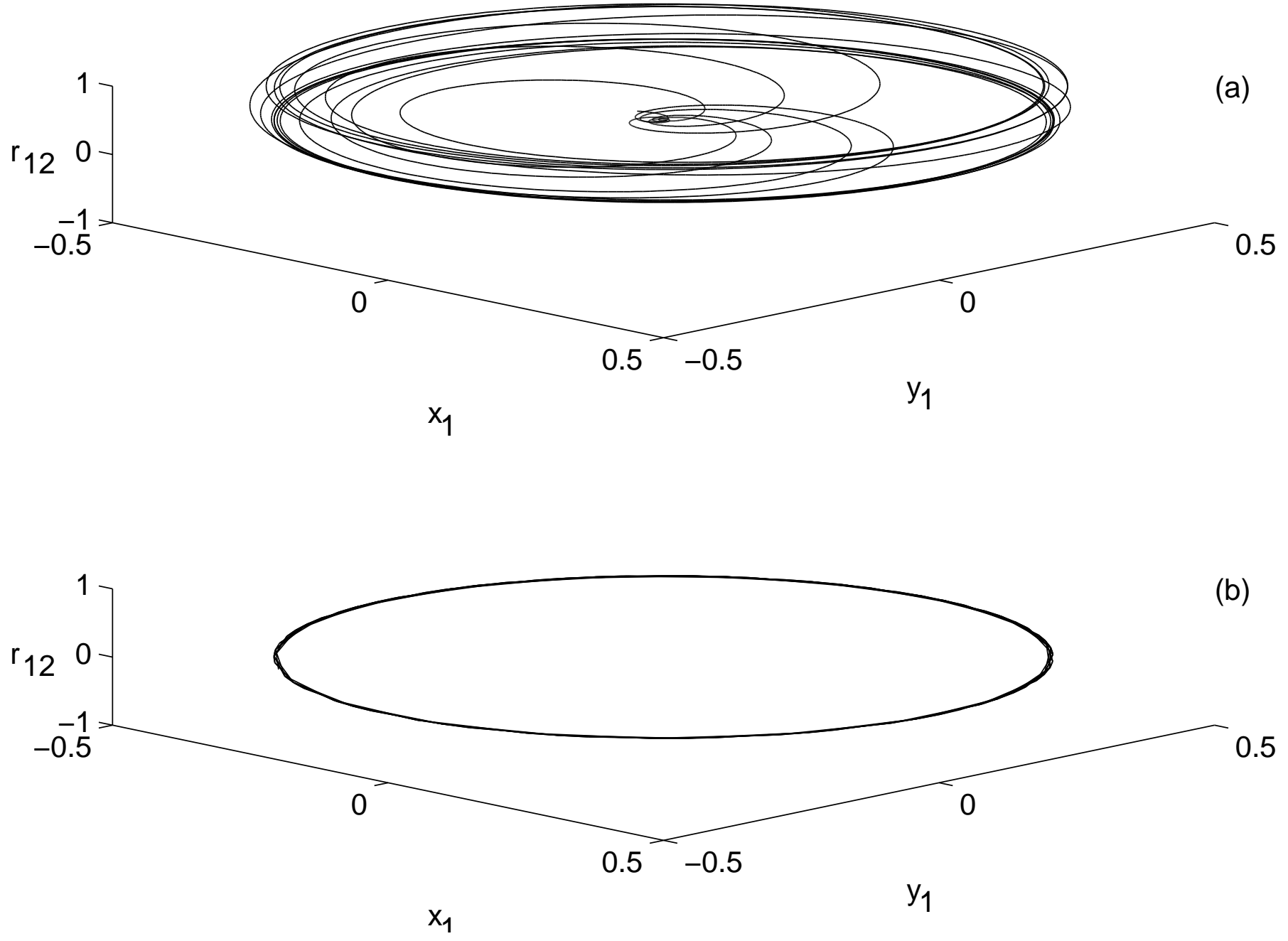